\title{
APPLICATION OF THE LATTICE BOLTZMANN METHOD TO THE FLOW PAST A SPHERE
}

\author{
Adam Kajzer, JaCeK Pozorski \\ Institute of Fluid-Flow Machinery, Polish Academy of Sciences, Gdańsk, Poland \\ e-mail: adkajzer@gmail.com; jp@imp.gda.pl
}

\begin{abstract}
The results of fully resolved simulations and large eddy simulations of bluff-body flows obtained by means of the Lattice Boltzmann Method (LBM) are reported. A selection of Reynolds numbers has been investigated in unsteady laminar and transient flow regimes. Computed drag coefficients of a cube have been compared with the available data for validation purposes. Then, a more detailed analysis of the flow past a sphere is presented, including also the determination of vortex shedding frequency and the resulting Strouhal numbers. Advantages and drawbacks of the chosen geometry implementation technique, so called "staircase geometry", are discussed. For the quest of maximum computational efficiency, all simulations have been carried out with the use of in-house code executed on GPU.
\end{abstract}

Keywords: bluff-body flow, Lattice Boltzmann Method, Large Eddy Simulation, GPU computing

\section{Introduction}

Computational Fluid Dynamics (CFD) is of higher and higher importance in science and engineering as it allows one to predict flow phenomena in investigated systems without carrying out experiments that tend to be increasingly costly and time consuming. The most popular CFD approach is the Finite Volume Method (FVM), see Versteeg and Malalasekera (2007), especially when the computational domain geometry is complex. The FVM is very well validated and has become an industrial standard in a wide range of applications. On the other hand, recent rapid developments in computer technology including Graphics Processing Units (GPU) and availability of high-level programming tools have made massive parallel computing relatively easy and inexpensive nowadays. This is an incentive to revisit the formulation of numerical schemes and algorithms.

The Lattice Boltzmann Method (LBM) is an alternative approach in CFD. Thanks to its explicit and local character (Succi, 2001), LBM is straightforward to parallelize, e.g., on the GPUs (Schoenherr et al., 2011). The important feature of the method is that it also allows easy handling of complex geometries. Satisfactory results obtained by means of LBM have been reported for various compressible and incompressible, turbulent, single- and multiphase flows (Arcidiacono et al., 2007; Chang et al., 2013; Prasianakis and Karlin, 2008; Pourmirzaagha et al., 2015). In a comprehensive paper by Hoelzer and Sommerfeld (2009), directly relevant for the present work, LBM was applied to the prediction of forces and moments acting on finite-size particles. Also, reactive flow phenomena including combustion, heat transfer and flows through porous media can be successfully simulated with the Lattice Boltzmann approach (Chiavazzo et al., 2010; Arcidiacono et al., 2008; Prasianakis and Karlin, 2007; Grucelski and Pozorski, 2015). Thus, it is worth to investigate different LBM approaches and implementation techniques to make it more and more accurate without sacrificing computational efficiency. 
In this paper, we present LBM simulations of a flow around a sphere in both laminar and turbulent regimes. The investigated case is well validated experimentally (Achenbach, 1972, 1974; Sakamoto and Haniu, 1990), so it is often treated as a benchmark for computational approaches (Jones and Clarke, 2008). The main aim of this work is to investigate LBM capability to predict macroscopic flow quantities (i.e. the drag coefficient and Strouhal number) for the "staircase geometry" scheme applied on a uniform lattice. This approach is the simplest possible way of spatial discretisation of the body geometry in LBM and allows one to efficiently parallelize the implementation of boundary conditions and evaluation of hydrodynamic forces. To the best of the authors' knowledge, the use of simplified geometry in the turbulent flow regime is a novel aspect of this work. Researchers investigated also more sophisticated methods, i.e. interpolation of the body boundary (Mei et al., 2002). Stiebler et al. (2011) presented an LBM simulation of the flow around a sphere with the use of local discretisation refinement. In the present work, simulations have been carried out for a selection of Reynolds numbers varying from 30 up to $10^{4}$. Large eddy simulation (LES) has been implemented according to the sub-grid scale (SGS) turbulence model of Smagorinsky (1963) that already had given good results in simulation of the turbulent Taylor-Green vortex (Kajzer et al., 2014).

\section{Lattice Boltzmann Method and implementation details}

\subsection{Fundamentals of the method}

The LBM is based on the kinetic theory of gases. The discretised Boltzmann equation is solved (instead of the Navier-Stokes equations) for discrete velocity distribution functions $f_{\alpha}(\mathbf{x}, t)$, in further sections also called populations. The spatial discretisation is done on a regular cubic grid; moreover, only a finite number of directions and magnitudes (indexed by $\alpha$ ) are allowed in the microscopic velocity field. In this paper, we present results obtained with a code implementing the $D 3 Q 15$ lattice scheme, i.e., $D=3$ dimensions and $Q=15$ allowed directions. Also popular, due to its better accuracy and only slightly higher computational cost, is the $D 3 Q 19$ model. All formulations and proofs of statements recalled in this Section can be found in the book of Succi (2001).

The discretised Boltzmann equation with the Bhatnagar-Gross-Krook closure for the collision operator takes the following form

$$
f_{\alpha}\left(\mathbf{x}+\Delta t \mathbf{e}_{\alpha}, t+\Delta t\right)-f_{\alpha}(\mathbf{x}, t)=\tau^{-1}\left(f_{\alpha}^{e q}(\mathbf{x}, t)-f_{\alpha}(\mathbf{x}, t)\right)
$$

where $\alpha$ is the index of the velocity direction $(\alpha=0,1, \ldots, Q-1), \tau$ is the nondimensional relaxation time and $\mathbf{e}_{\alpha}$ is the lattice velocity in the direction $\alpha$. The equilibrium distributions $f_{\alpha}^{e q}(\mathbf{x}, t)$, or more precisely $f_{\alpha}^{e q}(\rho(\mathbf{x}, t), \mathbf{u}(\mathbf{x}, t))$, corresponding to the density $\rho$ and macroscopic velocity $\mathbf{u}$ at the lattice node $\mathbf{x}$ at the time $t$, are calculated as follows

$$
f_{\alpha}^{e q}(\rho, \mathbf{u})=w_{\alpha} \rho\left(1+\frac{3}{c^{2}} \mathbf{e}_{\alpha} \cdot \mathbf{u}+\frac{9}{2 c^{4}}\left(\mathbf{e}_{\alpha} \cdot \mathbf{u}\right)^{2}-\frac{3}{2 c^{2}} \mathbf{u} \cdot \mathbf{u}\right)
$$

where $\rho=\sum_{\alpha=0}^{Q-1} f_{\alpha}$ is the fluid density, $\mathbf{u}=\rho^{-1} \sum_{\alpha=0}^{Q-1} f_{\alpha} \mathbf{e}_{\alpha}$ is the macroscopic fluid velocity, $w_{\alpha}$ is a weighting coefficient (depending on the lattice type $D n Q m$ ), $c=\Delta x / \Delta t$ is the lattice speed.

The discretised Boltzmann equation is solved in two steps, called respectively the collision step and the propagation step

$$
\begin{aligned}
& \widetilde{f}_{\alpha}(\mathbf{x}, t)=f_{\alpha}(\mathbf{x}, t)+\tau^{-1}\left(f_{\alpha}^{e q}\left(\mathbf{x}, t-f_{\alpha}(\mathbf{x}, t)\right)\right. \\
& f_{\alpha}\left(\mathbf{x}+\Delta t \mathbf{e}_{\alpha}, t+\Delta t\right)=\widetilde{f}_{\alpha}(\mathbf{x}, t)
\end{aligned}
$$


The explicit and local character of LBM is visible in the above equations: the collision step involves values of the flow fields (through $f^{e q}$ ) and populations at a single node only, and the propagation step consists in copying the post-collision populations to proper neighbouring nodes. In our implementation, $\Delta x=\Delta t=1$ which results in $c=1$. The pressure field is obtained from the following equation of state: $p=\rho c_{s}^{2}$, where the speed of sound is $c_{s}=c / \sqrt{3}$. It can be shown that $\mathbf{u}$ and $p$ satisfy the Navier-Stokes equations with the kinematic viscosity $\nu=(\tau-1 / 2) c_{s}^{2} \Delta t$ with an error $\mathcal{O}\left(\mathrm{Ma}^{2}\right)$.

\subsection{Boundary conditions}

As the discretised Boltzmann equation solves for the discrete velocity distribution functions, proper boundary conditions on these distributions have to be enforced to retrieve the physical behaviour of the fluid in the macroscopic sense (i.e., velocity and pressure). All types of boundary conditions used in presented simulations are widely described by Succi (2001). The easiest way to implement the immersed body geometry is to project it on regular lattice nodes. The lattice nodes are then marked with respective flags, "fluid" and "solid", say. That is why this variant is called "staircase geometry" (mesh-fitted surface of the body). Such an approach enables explicit use of the so-called bounce-back (BB) boundary condition. The populations outcoming from the solid nodes to the fluid nodes are replaced by populations moving in the opposite direction. This scheme makes the code efficient as it does not require any interpolation steps. Unfortunately, it is of the first order of accuracy in the described case (i.e. when the solid wall coincides with the lattice nodes). The inflow boundary condition corresponds to the constant velocity vector normal to the inflow plane, and the density (thus the pressure) is resultant. It is achieved by enforcing the populations to be in the equilibrium state $f_{\alpha}\left(\mathbf{x}_{i n}, t\right)=f_{\alpha}^{e q}\left(\mathbf{x}_{i n}, t\right)$ with $\mathbf{x}_{i n}$ being the inlet nodes, corresponding to the inflow velocity and the resulting density. The outflow boundary condition forces the density (and pressure) to be constant and the velocity gradient to vanish. It is realized by setting the populations in equilibrium related to the reference density $\rho_{0}$ and the velocity values in nodes preceding to the outflow plane in the normal direction $-\mathbf{e}_{n, \text { out }}$, pointing towards the domain interior: $f_{\alpha}\left(\mathbf{x}_{\text {out }}, t\right)=f_{\alpha}^{e q}\left(\mathbf{x}_{\text {out }}-\Delta t \mathbf{e}_{n, \text { out }}, t\right)$, where $\mathbf{x}_{\text {out }}$ are the outflow nodes. On the domain side boundaries, the symmetry plane boundary condition is imposed (this is achieved by mirror reflection of proper populations), and the density is resultant. Some other approaches to inflow and outflow conditions can be found in (Grucelski and Pozorski, 2013).

\subsection{Force evaluation}

Hydrodynamic forces acting on the sphere are calculated by means of the momentum exchange method. We decided to use this method although it is proposed for cases with boundary nodes lying exactly halfway beetwen the lattice nodes. Mei et al. (2002) raised some questions about the required body discretisation resolution as a function of the Reynolds number when using this method. It utilises only the advection and collision distribution functions $\left(f_{\alpha}\right.$ and $\left.\widetilde{f}_{\alpha}\right)$ without the necessity of computing the pressure and shear stress. The total force $\mathbf{F}$ acting on the immersed body is calculated as follows

$$
\mathbf{F}=\sum_{\mathbf{x}_{s f}} \sum_{\alpha}\left(f_{-\alpha}\left(\mathbf{x}_{s f}\right)+\widetilde{f}_{\alpha}\left(\mathbf{x}_{s f}\right)\right) \mathbf{e}_{\alpha}
$$

where $\mathbf{x}_{s f}$ denotes the fluid nodes that have at least one solid neighbour, and the subscript $-\alpha$ is defined by $\mathbf{e}_{-\alpha}=-\mathbf{e}_{\alpha}$. 


\subsection{Large Eddy Simulation in LBM}

LES is a method of turbulence modelling that resolves spatially filtered flow fields (Aubard et al., 2013; Sagaut and Grohens, 1999). The method can be implemented as a local increase of the kinematic viscosity $\nu$

$$
\nu_{\text {eff }}=\nu+\nu_{\text {sgs }}
$$

where $\nu_{\text {eff }}$ is the effective local viscosity and $\nu_{\text {sgs }}$ denotes the turbulent (or sub-grid scale, SGS) viscosity which in the model of Smagorinsky (1963) is computed as

$$
\nu_{\text {sgs }}=\left(C_{S} \Delta\right)^{2}|S|
$$

where $\Delta$ is the filter size, $|S|=\sqrt{2 S_{i j} S_{i j}}$ and $S_{i j}=\frac{1}{2}\left(\frac{\partial u_{i}}{\partial x_{j}}+\frac{\partial u_{j}}{\partial x_{i}}\right)$ is the strain rate tensor, $C_{S}$ is a constant, most often equal to 0.17 .

We present now the application of the Smagorinsky SGS model to LBM (Chang et al., 2013; Stiebler et al., 2011). In the lattice Boltzmann method, the kinematic viscosity of the fluid $\nu$ is uniquely linked with the relaxation time $\tau$. The effective relaxation time is determined as

$$
\tau_{\text {eff }}=3\left(\nu+\nu_{\text {sgs }}\right) \frac{\Delta t}{(\Delta x)^{2}}+\frac{1}{2}
$$

It can be shown that

$$
\nu_{\text {sgs }}=\left(C_{S} \Delta\right)^{2}|S|=\frac{1}{6}\left(\sqrt{\tau^{2}+\frac{18\left(C_{S} \Delta\right)^{2}|P|}{\rho}}-\tau\right)
$$

where $|P|=\sqrt{2 P_{i j} P_{i j}}$ with $P_{i j}=\sum_{\alpha} e_{\alpha i} e_{\alpha j}\left(f_{\alpha}-f_{\alpha}^{e q}\right)$ being the stress tensor. Combining Eqs. (2.7) and (2.8), we obtain

$$
\tau_{\text {eff }}=\frac{\tau}{2}\left(1+\sqrt{1+\frac{18\left(C_{S} \Delta\right)^{2} P}{\rho \tau^{2}}}\right)
$$

In our implementation of LES in the LBM, we set $\Delta=\Delta x$.

\section{Flow cases and results}

\subsection{Validation case: flow past a cube}

In order to validate the computer implementation, we made a simulation of the flow around a cube, as the solid boundary is exactly modelled within the "staircase geometry" approach. All computations have been carried out with the use of an in-house LBM code written in NVIDIA CUDA-C language and executed on NVIDIA GeForce series GPU. The code does not involve external libraries or user-defined data structures - only CUDA built-in types are used. The spatial resolution was set to $644 \times 244 \times 244$ nodes in the $x, y$ and $z$ directions respectively, resulting in about 38 million of nodes which exploited all available GPU memory (6 GB). The cube centre was placed at $x=160$ l.u. (lattice units) and the cube edge was 32 l.u. Setting the inflow speed to 0.0289 l.u. resulted in $\mathrm{Ma}<0.1$ (while the lattice sound speed $c_{s} \approx 0.57$ ) in the whole domain so the flow could be treated as incompressible. The computations were carried out at Reynolds numbers $\mathrm{Re}=50,100,200,300$ (fully resolved simulations) and $10^{4}$ (LES), where $\operatorname{Re}=U_{i n} D / \nu=3 U_{l} N /(\tau-0.5)$ with $D$ being the cube edge size (characteristic length) in physical units, where $U_{i n}$ is the inlet speed in physical units, $U_{l}$ is the inlet speed in lattice units, 


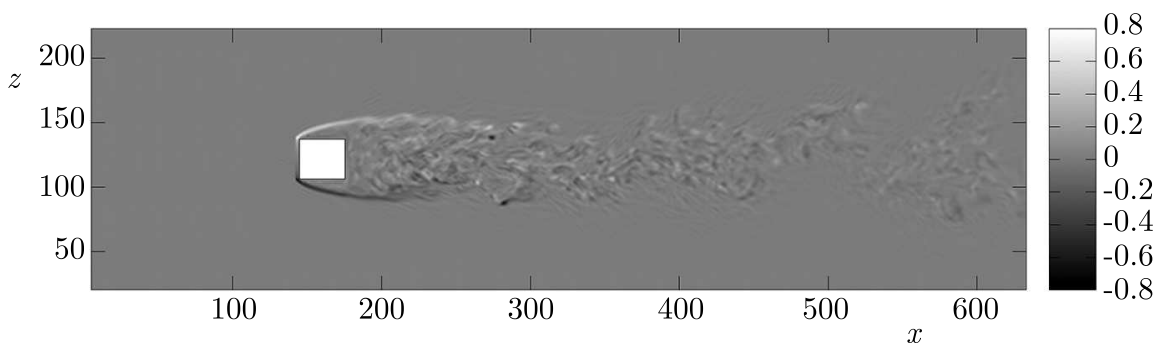

Fig. 1. A snapshot of the vorticity $y$-component in the flow past a cube at $\operatorname{Re}=10^{4}$ (cross-section normal to the $y$-axis in the symmetry plane, $t^{+}=95$ )

$N$ is the number of lattice nodes per cube characteristic length. The obtained $y$ component of vorticity field at $\operatorname{Re}=10^{4}$ at non-dimensional time $t^{+}=95$ (statistically steady state), where $t^{+}=t U_{l} / N$ with $t$ being the time in l.u., is shown in Fig. 1.

In Fig. 2, the results of the computed drag coefficient $C_{D}$ are shown. It is calculated as $C_{D}=2 F_{x} / \rho U_{l}^{2} S$, where $F_{x}$ is the force acting on the cube in the $x$-direction, see Eq. (2.4), $\rho$ is the density, $U_{l}$ is the inflow speed in l.u. and $S$ is the cross-section area of the cube in l.u. At $\operatorname{Re}=50,100,200$ and 300, the computed drag is in very good agreement with the data reported by Saha (2004) obtained by means of the MAC (Marker And Cell) method. His results were summarised in the form of correlation $C_{D}=(24 / \mathrm{Re})\left(1+0.232 \cdot \mathrm{Re}^{0.628}\right)$. For Reynolds numbers above 300 in the transient flow regime, some scattered data on the drag coefficient are available, but no precise values or correlations are given (Hoelzer and Sommerfeld, 2009). In the turbulent regime, at $\mathrm{Re}=10^{4}$, the drag coefficient is slightly overestimated in comparison to the Re-independent value $C_{D}=1.05 \pm 0.05$ given by Holmes et al. (2004). The reason of this discrepancy is discussed in the next Section.

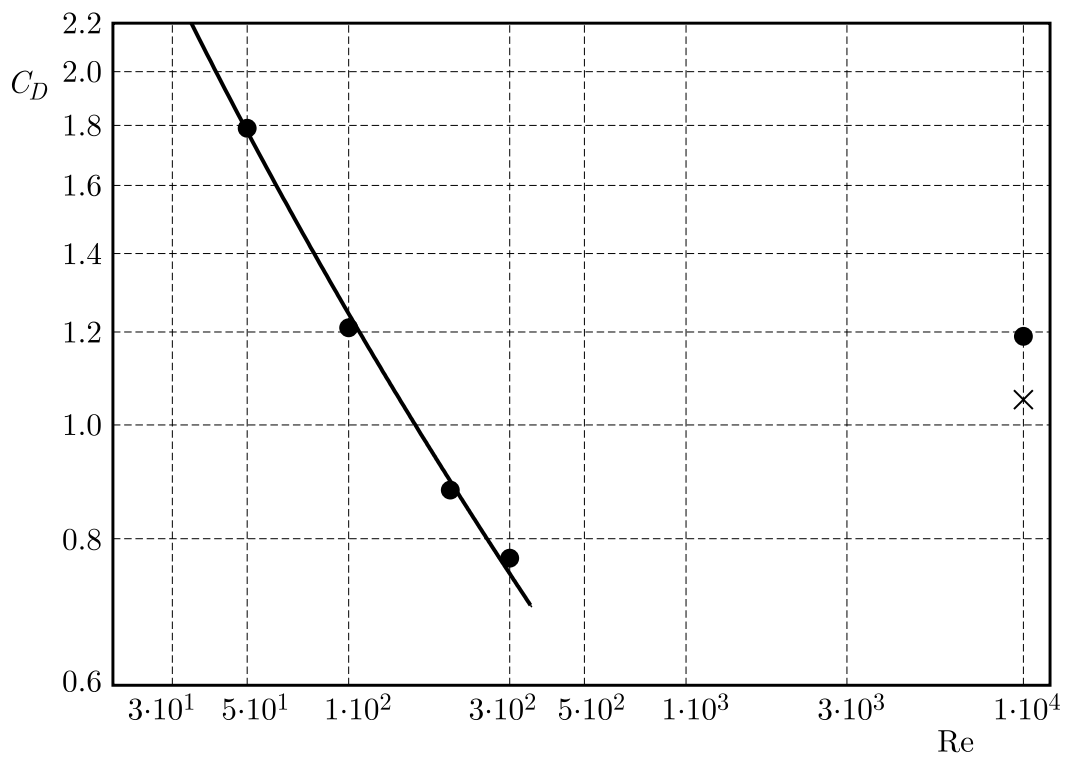

Fig. 2. Drag coefficient of cube; $\bullet$ : present LBM results; ——: approximation of numerical results given by Saha (2004) for laminar flow; $\times: C_{D}=1.05 \pm 0.05$, valid for the turbulent regime

\subsection{Flow past a sphere}

After the validation tests presented above, simulations of the flow past a body of simplest curvilinear geometry - a sphere - have been carried out. The sphere diameter was set to 32 lattice units which resulted in domain blockage about $1.3 \%$ (defined as the ratio of cross-stream areas 
of the sphere and the domain). All other parameters were set to the same values as in the simulations of the flow past a cube. Computations without turbulence modelling at $\mathrm{Re}=30$, 50, 100, 300 and 500 were performed. In Table 1, the computed drag coefficients are compared with the experimental correlation reported by Clift et al. (1978). The computed drag coefficients are in good agreement with the experimental results up to $\mathrm{Re}=300$ (see Fig. 3). At $\operatorname{Re}=500$, the error is more significant, which suggests that the simulation is underresolved. Therefore, at higher Reynolds numbers $\left(10^{3}, 3 \cdot 10^{3}\right.$ and $10^{4}$ ), the LES model (described in Sec. 2.4) was used. In Fig. 4, the map of the ratio of the turbulent-to-molecular viscosity $\nu_{\text {sgs }} / \nu$ is shown at $\operatorname{Re}=10^{3}$. It is clearly visible that the resolution used in these simulations is barely sufficient since the maximum values of the SGS viscosity are comparable to the molecular viscosity. At $\operatorname{Re}=10^{4}$, the values of $\nu_{\text {sgs }}$ can locally be even one order of magnitude higher than $\nu$ in the vicinity of the sphere surface, which means that the boundary layer is not adequately resolved. Arguably, this explains the overestimation of the drag coefficient. Moreover, since we are close to the stability limit of computations, some artefacts are visible in the upstream region in Fig. 4 due to the weakly compressible nature of LBM. Computations at higher Reynolds numbers (above $10^{4}$, not shown in Fig. 3 ) reveal that the drag coefficient remains almost constant. This effect is expected since capturing these slight differences, in particular a correct prediction of the drag crisis at $\operatorname{Re} \sim 2 \cdot 10^{4}$, would require very fine meshes (Rodriguez et al., 2013).

Table 1. Computed and experimental drag coefficients $C_{D}$ of the sphere. At $\operatorname{Re}=10^{3}$ and above, the LES approach has been applied

\begin{tabular}{|c|c|c|}
\hline Re & exp. data & LBM (rel. error in \%) \\
\hline \hline 30 & 2.12 & $2.08(1.9 \%)$ \\
\hline 50 & 1.57 & $1.55(1.3 \%)$ \\
\hline 100 & 1.09 & $1.08(1.0 \%)$ \\
\hline 300 & 0.65 & $0.67(3.1 \%)$ \\
\hline 500 & 0.55 & $0.59(7.3 \%)$ \\
\hline $10^{3}(\mathrm{LES})$ & 0.47 & $0.55(17 \%)$ \\
\hline $3 \cdot 10^{3}(\mathrm{LES})$ & 0.40 & $0.53(33 \%)$ \\
\hline $10^{4}(\mathrm{LES})$ & 0.41 & $0.54(32 \%)$ \\
\hline
\end{tabular}

The frequency spectra of velocity in the wake area have also been calculated in order to obtain the Strouhal number defined as

$$
\mathrm{St}=\frac{f_{0} D}{U_{\text {in }}}
$$

where $f_{0}$ denotes the frequency of vortex shedding, $D$ is the sphere diameter and $U_{i n}$ is the inflow velocity. The frequency spectra of the cross-stream velocity components were obtained by means of FFT. The velocity probes were placed at $\mathbf{x}=\mathbf{x}_{c}+x_{p} \mathbf{x}_{0}+y_{p} \mathbf{y}_{0}+z_{p} \mathbf{z}_{0}$, where $\mathbf{x}_{c}$ denotes the position of the sphere centre, $\mathbf{x}_{0}, \mathbf{y}_{0}, \mathbf{z}_{0}$ are axes unit vectors, and $x_{p}=3.0 D$, $y_{p}=0.3 D$ and $z_{p}=0.5 D$. The spectra were computed at $\operatorname{Re}=10^{3}$ and $\operatorname{Re}=10^{4}$. Figure 5 shows the computed spectra of the $y$-component of velocity, $F\left[u_{y}\right]$, as a function of the dimensionless frequency $f^{*}=f D / U_{i n}$.

The obtained results match quite well the experimental data at $\mathrm{Re}=10^{3}$. Sakamoto and Haniu (1990) reported $\mathrm{St}=0.18-0.20$ with $\sim 4 \%$ measurement errors, while the present LBM result is $\mathrm{St}=0.19$. At $\mathrm{Re}=10^{4}$, the spectrum has significant amplitudes also at higher frequencies, which is an expected result as the wake becomes more turbulent than at $\operatorname{Re}=10^{3}$. Moreover, it is hard to distinguish a clearly dominating frequency in the neighbourhood of $f^{*}=0.2$ (obtained in experiments), although the highest value is achieved at $f^{*}=0.17$. This result should however be taken with care since the simulation is underresolved. 


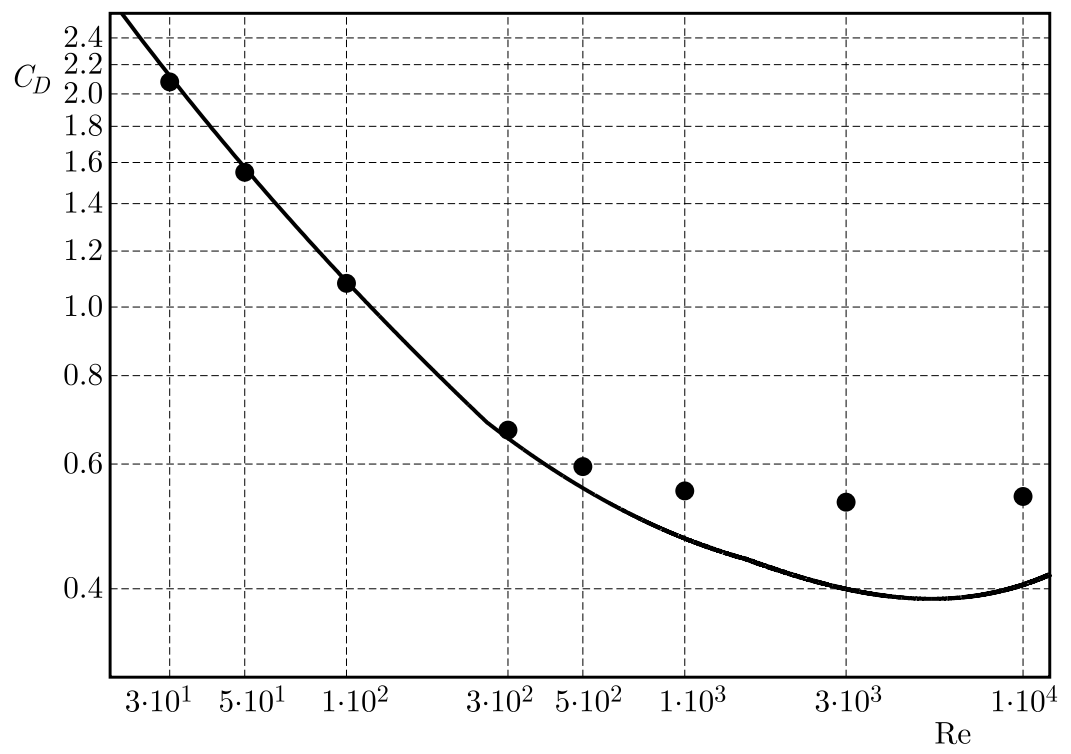

Fig. 3. Drag coefficient of the sphere. •: present LBM computations; — $—$ : approximation of experimental results according to Clift et al. (1978)

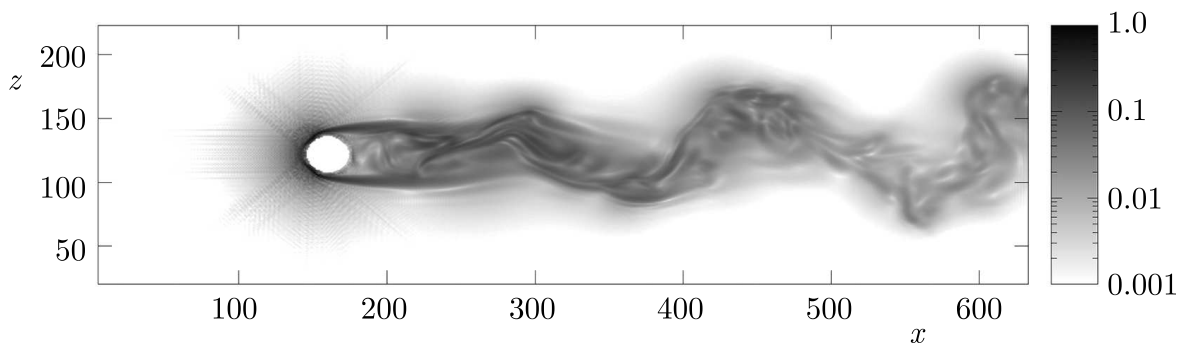

Fig. 4. The grayscale map of $\nu_{\text {sgs }} / \nu$ at $\mathrm{Re}=10^{3}$ at non-dimensional time $t^{+}=95$
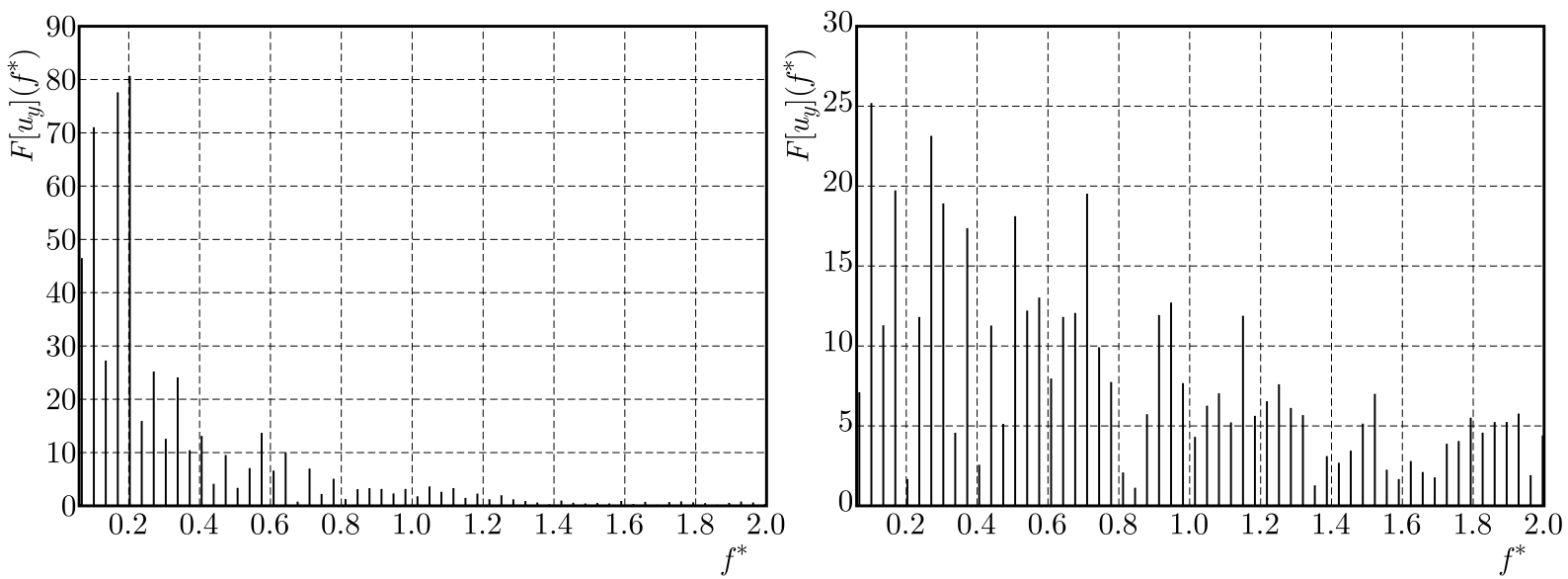

Fig. 5. The spectrum of the $y$-component of velocity obtained in LBM simulations expressed in terms of the dimensionless frequency $f^{*}$ at $\operatorname{Re}=10^{3}$ (left) and $\operatorname{Re}=10^{4}$ (right)

\section{Conclusions and perspectives}

The methods presented in this paper allow one to accurately predict the flow past simple bluff bodies in terms of the drag force and the Strouhal number in the range of low and moderate Reynolds numbers. In the transitional and turbulent regimes, we have added the LES closure for non-resolved flow scales. In the case of a turbulent flow, it would be however necessary to 
introduce a local lattice refinement to solve the boundary layer and wake with sufficient accuracy. Further validation of the presented methods should involve study of more complex phenomena in bluff-body flows, e.g. wake dynamics and structures which were investigated in an experimental way by Klotz et al. (2014) and Szaltys et al. (2011).

The presented implementation of boundary conditions, force evaluation and turbulence model do not deprive LBM from its explicit local character, so the problem size (the number of lattice nodes $N$, say) does not have a big impact on the total simulation time as the computational complexity remains of $\mathcal{O}(N)$. These features of LBM make it incomparably faster than other methods, even up to two orders of magnitude faster than FVM executed on a single multicore CPU of comparable cost with a similar programming effort (Kajzer et al., 2014). Introduction of the block-wise refinement will not require modification of the presented approaches. However, it will make the implementation slightly more complicated in comparison to the homogenous lattice.

Summarising, the Lattice Boltzmann Method seems to be a promising tool for external flow computations in a wide range of Reynolds numbers.

Acknowledgments

The authors would like to thank Dr Arkadiusz Grucelski (IMP PAN Gdańsk) and an unknown Referee for their insightful remarks and helpful suggestions.

\section{References}

1. Achenbach E., 1972, Experiments on the flow past spheres at very high Reynolds numbers, Journal of Fluid Mechanics, 54, 565-575

2. AChenbach E., 1974, Vortex shedding from sphere, Journal of Fluid Mechanics, 62, 209-221

3. Arcidiacono S., Mantzaras J., Karlin I.V., Frouzakis C., 2007, Lattice Boltzmann method for the simulation of multi-component mixtures, Physical Review E, 76, 046703

4. Arcidiacono S., Mantzaras J., Karlin I.V., 2008, Lattice Boltzmann method for the simulation of catalytic reactions, Physical Review E, 78, 046771

5. Aubard G., Volpiani P.S., Gloerfelt X., Robinet J.C., 2013, Comparison of subgrid-scale viscosity models and selective filtering strategy for large-eddy simulations, Flow, Turbulence and Combustion, 91, 497-518

6. Chang S.C., Yang Y.T., Chen C.K., Chen W.L., 2013, Application of the lattice Boltzmann method combined with large-eddy simulations to turbulent convective heat transfer, International Journal of Heat and Mass Transfer, 66, 338-348

7. Chiavazzo E., Karlin I.V., Gorban A.N., Boulouchos K.B., 2010, Coupling of the model reduction technique with the lattice Boltzmann method for combustion simulations, Combustion and Flame, 157, 1833-1849

8. Clift R., Grace J. R., Weber M.E., 1978, Bubbles, Drops, and Particles, Academic, New York

9. Grucelski A., Pozorski J., 2013, Lattice Boltzmann simulations of flow past an obstacle and in simple porous media, Computers and Fluids, 71, 406-416

10. Grucelski A., Pozorski J., 2015, Lattice Boltzmann simulations of heat transfer in flow past a cylinder and in simple porous media, International Journal of Heat and Mass Transfer, 86, 139-148

11. Hoelzer A., Sommerfeld M., 2009, Lattice Boltzmann simulations to determine drag, lift and torque acting on non-spherical particles, Computers and Fluids, 38, 572-589

12. Holmes J., English E., Letchford C., 2004, Aerodynamic forces and moments on cubes and flat plates, with applications to wind-borne debris, Fifth International Colloquim on Bluff Body Aerodynamics and Applications, Ottawa, Canada, 11-15 July 2004 
13. Jones D.A., Clarke D.B., 2008, Simulation of Flow Past a Sphere using the Fluent Code, Defence Science and Technology Organisation, Maritime Platforms Division, Victoria, Australia

14. Kajzer A., Pozorski J., Szewc K., 2014, Large-eddy simulations of 3D Taylor-Green vortex: comparison of Smoothed Particle Hydrodynamics, Lattice Boltzmann and Finite Volume methods, Journal of Physics: Conference Series, 530, 012019

15. Klotz L., Goujon-Durand S., Rokicki J., Wesfreid J.E., 2014, Experimental investigation of flow behind a cube for moderate Reynolds numbers, Journal of Fluid Mechanics, 750, 73-98

16. Mei R., Yu D., Shyy W., Luo L.-S., 2002, Force evaluation in the lattice Boltzmann method involving curved geometry, Physical Review E, 65, 0412037

17. Pourmirzangha H., Afrouzi H.H., Mehrizi A.A., 2015, Nano-particles transport in a concentric annulus: a Lattice-Boltzmann approach, Journal of Theoretical and Applied Mechanics, 53, 683-695

18. Prasianakis N., Karlin I.V., 2007, Lattice Boltzmann simulation of thermal flows on standard lattices, Physical Review E, 76, 016702

19. Prasianakis N., Karlin I.V., 2008, Lattice Boltzmann simulation of compressible flows on standard lattices, Physical Review E, 78, 016704

20. Rodriguez I., Lehmkuhl O., Borrell R., Paniagua L., Perez-Segarra C.D., 2013, High performance computing of the flow past a circular cylinder, Procedia Engineering, 63, 166-172

21. Sagaut P., Grohens R., 1999, Discrete filters for large eddy simulation, International Journal of Numerical Methods in Fluids, 31, 1195-1220

22. SaнA A.K., 2004, Three-dimensional numerical simulations of the transition of flow past a cube, Physics of Fluids, 16 5, 1630-1646

23. Sakamoto H., Haniu H., 1990, A study on vortex shedding from spheres in a uniform flow, Journal of Fluids Engineering, 112, 4, 386-392

24. Schlichting H., 1979, Boundary-Layer Theory, 7th ed., McGraw-Hill, New York

25. Schoenherr M., Kucher K., Geier M., Stiebler M,. Freudiger S., KrafczyK M., 2011, Multi-thread implementations of the lattice Boltzmann method on non-uniform grids for CPUs and GPUs, Computers and Mathematics with Applications, 61, 3730-3743

26. Smagorinsky J., 1963, General circulation experiments with the primitive equations, Monthly Weather Review, 91, 3, 99-164

27. Stiebler M., Krafczyk M., Freudiger S., Geier M., 2011, Lattice Boltzmann large eddy simulation of subcritical flows around a sphere on non-uniform grids, Computers and Mathematics with Applications, 61, 3475-3484

28. Succi S., 2001, The Lattice Boltzmann Method for Fluid Dynamics and Beyond, Clarendon Press, Oxford

29. Szaltys P., Chrust M., Przadka A., Goujon-Durand S., Tuckerman L.S., Wesfreid J.E., 2011, Nonlinear evolution of instabilities behind spheres and disks, Journal of Fluids and Structures, 28, 483-487

30. Versteeg H.K., Malalasekera W., 2007, An Introduction to Computational Fluid Dynamics: the Finite Volume Method, Pearson Education Ltd., Harlow, England, New York 